\title{
SHiP project as a new facility at intensity frontier
}

\author{
V.Shevchenko ${ }^{1,2, *}$, on behalf of the SHiP Collaboration. \\ ${ }^{1}$ National Research Centre "Kurchatov Institute" ac.Kurchatova sq., 1, Moscow 123182 Russia \\ ${ }^{2}$ Far Eastern Federal University, Sukhanova str. 8, Vladivostok 690950 Russia
}

\begin{abstract}
.
SHiP (Search for Hidden Particles) is a new general purpose fixed target facility suggested for SPS accelerator at CERN. The experiment is currently in Comprehensive Design Report preparation phase, aiming to become a part of European Strategy for Particle Physics update in 2019. In the initial phase of SHiP, the $400 \mathrm{GeV}$ proton beam extracted from the SPS will be dumped on a heavy target producing $2 \times 10^{20}$ protons on target in 5 years. A dedicated detector, based on a long vacuum tank followed by a spectrometer and particle identification detectors, will allow probing a variety of models with light long-lived exotic particles and masses below $O(10) \mathrm{GeV} / \mathrm{c}^{2}$. The main focus will be the physics of the so-called Hidden Portals, i.e. search for Dark Photons, Light scalars and Heavy Neutrinos. The sensitivity to Heavy Neutrinos will allow for the first time to probe, in the mass range between the kaon and the charm meson mass, a coupling range for which Baryogenesis and active neutrino masses could be explained simultaneously. Another dedicated detector will allow the study of tau-neutrino physics.
\end{abstract}

\section{Introduction}

Current situation in particle physics is characterized by undisputable triumph of the Standard Model (SM). The Higgs boson is discovered, many highly nontrivial SM effects are confirmed, and no a single sign of new particles or effects beyond the SM has been seen. Many suggested scenarios of New Physics phenomena in TeV ballpark, such as supersymmetry, extra dimensions, compositeness etc are ruled out or at least strongly constrained.

On the other hand, there are no doubts that physics beyond the SM does exist. Neutrino masses and oscillations; dark matter; baryon asymmetry of the Universe show that clearly. It should not be forgotten that there are many unanswered «why» and «how» in the SM itself: how is EW scale so smaller than UV scale? why hierarchy between SM scales? why are the roles of doublets and singlets so different? why 3 generations? why CKM hierarchy? why $\mathrm{CP}$ ? etc.

From more theoretical prospective, the SM is known to be not a self-consistent field theory. Landau pole, anomalies, naturalness issues mean that the SM cannot be considered as the ultimate theoretical framework. On the other hand, it is well known that things in the SM are arranged in such a way, that (almost) all the above mentioned problems are either cured or physically irrelevant. A good example is anomaly cancellation between quark and

*e-mail: Vladimir.Shevchenko@cern.ch 
lepton sectors, which would not be possible without delicate SM charge distribution between particles.

It is commonly believed that the main discovery of Large Hadron Collider (LHC) is the Higgs boson. However, much more important is not the fact of the Higgs boson existence but where it does exist. To our view, the most important finding of LHC is the fact that Higgs boson and top quark masses correspond to metastability region of the SM vacuum [1], see Fig.1. The ideas around that has been discussed for decades by many authors, notably by I. V. Krive, A. D. Linde, N. Cabibbo, L. Maiani, G. Parisi, R. Petronzio, M.Lindner, H.B.Nielsen, C.Froggatt, J. Elias-Miro, J. R. Espinosa, G. F. Giudice, G. Isidori, A. Riotto, A. Strumia, J. R. Espinosa, M. Quiros, G.Altarelli. It is very suggestive that historically the things went the other way around: the Higgs boson and top quark masses were predicted in the paper by C.Froggatt and H.B.Nielsen [2] back in 1995 assuming the SM vacuum is metastable (in the framework of the so called "multiple point principle"). The crucial assumption for this prediction to be credible is the absence of new (heavy) degrees of freedom which are nonsinglets with respect to the SM interactions. In other words, this is a strong indication to no New Physics up to the Planck scale.

This apparent paradox is perhaps a hint that it is more useful to think not about New Physics (NP) that could solve theoretical problems of the SM, but about NP that could explain observed effects beyond the SM (in hope that theory will settle things anyway). Taking this point of view we can easily come to the conclusion that such NP would not necessary be new heavy degrees of freedom, we are looking for at LHC experiments on energy frontier. It could well be that new particles are relatively light but so feebly interacting that they have escaped direct experimental detection so far. A typical example of models of this type is given by $v \mathrm{MSM}$ model [3], where the role of new degrees of freedom is played by right partners of the SM neutrino. The lightest particle, $N_{1}$, has mass in keV range and serves as a dark matter candidate while the heavier particles $N_{2}$ and $N_{3}$ are responsible for neutrino masses (via seesaw mechanism) and baryon asymmetry (via leptogenesis). Their masses are not predicted by the model but can well be in a few $\mathrm{GeV}$ range.

In more general terms one can speak in terms of the portals to the SM [4]. The total Lagrangian has the following form:

$$
L=L_{S M}+L_{H S}+L_{\text {Portal }}
$$

where $L_{S M}$ is the SM Lagrangian, Hidden Sector (HS) contains Dark Matter and can have very complicated structure, while $L_{\text {Portal }}$ is made of terms responsible for interaction between HS particles and some singlet operator built from SM fields. Most work has been done up to now on renormalizable portals (scalar, vector and neutrino), see references in [4]. Of particular interest is the $v$ MSM model [3] with the Largangian

$$
L=L_{S M}+\bar{N}_{I} i \partial_{\mu} \gamma^{\mu} N_{I}-F_{\alpha I} \Phi \bar{N}_{I} L_{\alpha}-\frac{M_{I}}{2} \bar{N}_{I}^{c} N_{I}+\text { h.c. }
$$

The new particles - Heavy Neutral Leptons $N_{2}$ and $N_{3}$ are among the objects to search for at the SHiP facility.

It is clear that there are two crucial requirements one has to obey for experimental search of such particles: huge initial intensity and large (to be more precise, very long) controlled detector volume. A project of experimental facility capable to do the job is described below.

\section{The SHiP Project: overview}

SHiP (Search for Hidden Particles) [4, 5] is a new general purpose fixed target facility proposed at the CERN SPS accelerator to search for particles predicted by Hidden Portals. These 
particles are expected to be predominantly accessible through the decays of heavy hadrons. The facility is therefore designed to maximise the production and detector acceptance of charm and beauty mesons, while providing the cleanest possible environment. The $400 \mathrm{GeV}$ proton beam extracted from the SPS will be dumped on a high density target with the aim of accumulating $2 \times 10^{20}$ protons on target during 5 years of operation. The charm production at SHiP exceeds any existing and planned facility. The experiment is supposed to be located in the North Area and shares TT20 transfer line and the slow extraction mode with other fixed target programmes.

A general layout is depicted on Fig.2. Water cooled target will dissipate $4 \times 10^{13}$ protons at $7 \mathrm{sec}$ which gives average power $355 \mathrm{~kW}$ (2.56 MW during $1 \mathrm{sec}$ spill). Initial dose is estimated to be around $50 \mathrm{~Sv} / \mathrm{h}$. The target is segmented: high-Z hybrid solution is composed of molybdenum alloy strips and pure tungsten ones.

The main decay vessel (in vacuum option) has the size $10 \times 5 \times 60$ meters. Liquid or plastic scintillator options for veto system (with the readout by optical modules) are being considered. A dedicated detector, based on a long vacuum tank followed by a spectrometer and particle identification detectors, will allow probing a variety of models with light long-lived exotic particles and masses below $O(10) \mathrm{GeV} / \mathrm{c}^{2}$. Since the hidden sector particles originating from charm and beauty are produced with a significant transverse momentum with respect to the beam axis, the detector should be placed as close as possible to the target. A critical component of SHiP is the muon shield, which deflects the high flux of muons produced in the target, that would represent a very serious background for the particle searches, away from the detector [6], see Fig.3. The detector is designed to fully reconstruct the exclusive decays of hidden particles and to reject the background down to below 0.1 events in the sample of $2 \times 10^{20}$ protons on target. The spectrometer is to accurately reconstruct the decay vertex, mass and impact parameter of the decaying particle at the target. A set of calorimeters followed by muon chambers provide identification of electrons, photons, muons and charged hadrons. A dedicated timing detector measures the coincidence of the decay products, which allows the rejection of combinatorial backgrounds. The decay volume is surrounded by background taggers to tag neutrino and muon inelastic scattering in the surrounding structures, which may produce long-lived $\mathrm{SM}$ particles, such as $\mathrm{K}_{\mathrm{L}}$, that have similar topologies to the expected signals. The overall scheme of the sub-detectors is depicted on Fig.4.

The SHiP experimental facility will also be suited for studying interactions of tau neutrinos. It will host an emulsion cloud chamber based on the OPERA detector concept, upstream of the hidden-particle decay volume, followed by a muon spectrometer, see Fig.5. For the total number of neutrino one can estimate $N_{\nu_{\tau}+\bar{v}_{\tau}} \sim N_{\text {pot }} \times 3 \times 10^{-5}$. This will exceed the statistics of DONUT and CHARM experiments by 3-5 orders of magnitude.

The very important property of the models like $v \mathrm{MSM}$ is that they constrain the interactions of the new particles with the SM ones from above as well as from below. Indeed, if the interaction was too weak, the see-saw mechanism for giving masses to neutrinos would not work. If, on the contrary, the interaction was too strong, the baryon asymmetry would be washed out in the course of cosmological evolution (see detailed discussion and references in [4]). Therefore the typical profile of the allowed region in coupling-mass coordinates is a kind of strip, and the SHiP experiment can dig a sizeable fraction of it up to a certain mass (and, in ideal case, "to touch" the lower bound), see Fig.6, where it is schematically shown. Of course, to get the detailed sensitivity plots a full scale Monte-Carlo simulation is needed. Some examples for various portals are depicted on Figs. 7, 8, 9 (from [7]). 

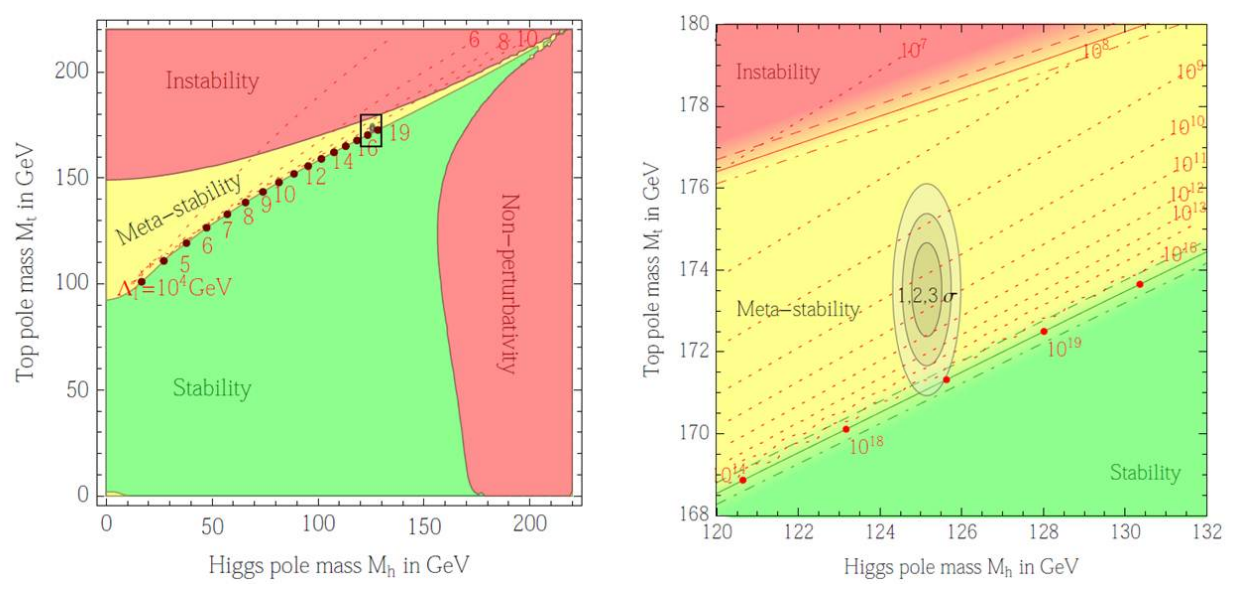

Figure 1. Meta-stability of the SM vacuum (from [1]).

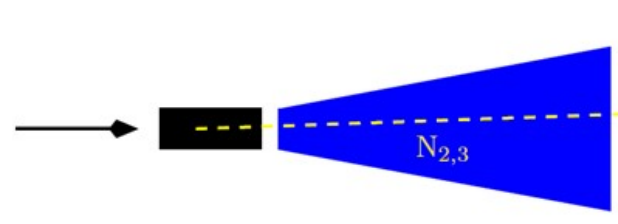

$p$-beam Target $\quad \mu$-shield

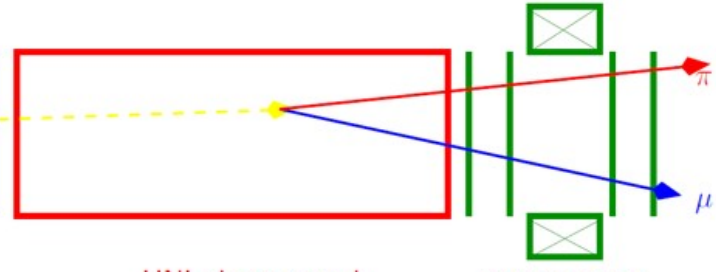

HNL decay vessel

spectrometer

Figure 2. General layout of the SHiP experiment

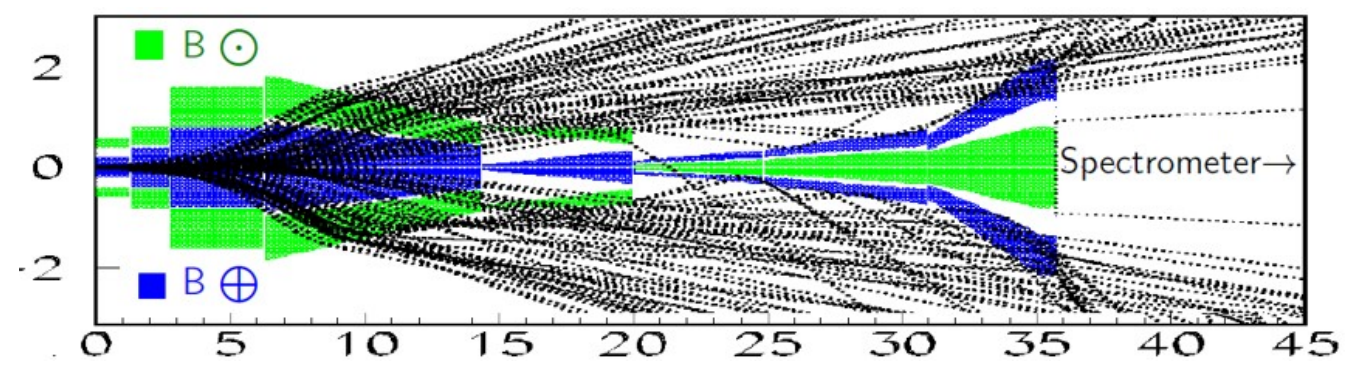

Figure 3. Muon trajectories in the magnetic shield 


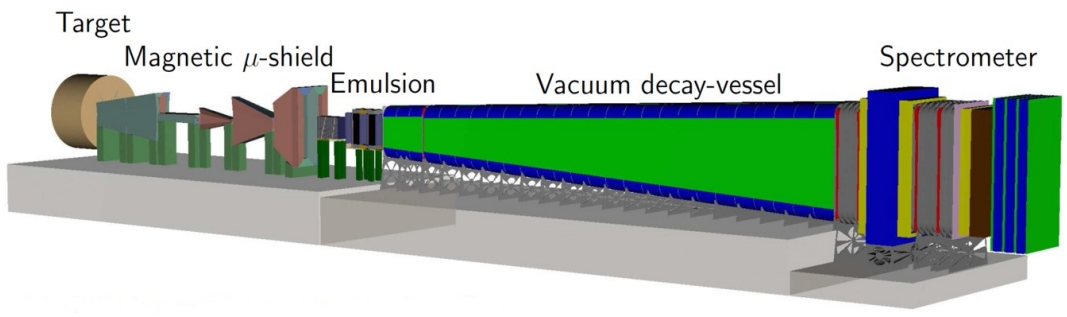

Figure 4. Detector subsystems of the SHiP experiment

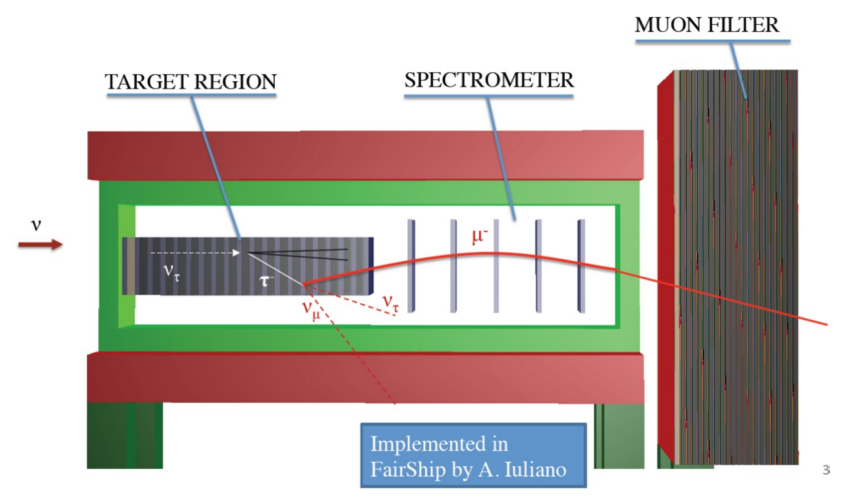

Figure 5. Neutrino detector subsystem of the SHiP experiment
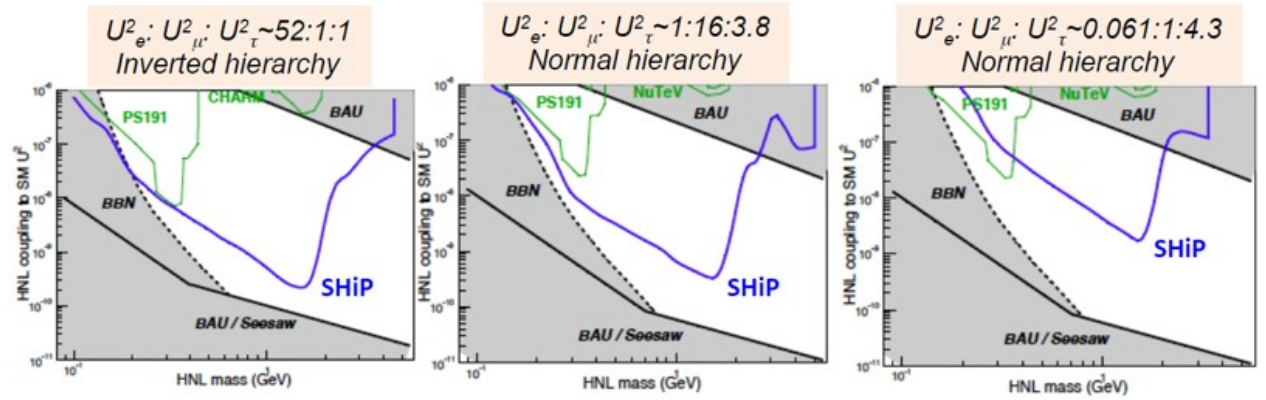

Figure 6. General structure of SHiP sensitivity plots (to HNL particles)

\section{Conclusions}

No new heavy particles have been discovered by ATLAS and CMS experiments (leaving aside the $126 \mathrm{GeV}$ Higgs boson) for 9 years of LHC running. Indirect searches of NP by LHCb and other precision experiments provided very high, hundreds-TeV bounds for heavy NP degrees of freedom in some channels (with no flavor alignment). On the other hand the experimentally known NP phenomena (neutrino oscillations, dark matter etc) give no indication to the intrinsic scales of the corresponding physics. From theoretical side, all that calls for reconsid- 


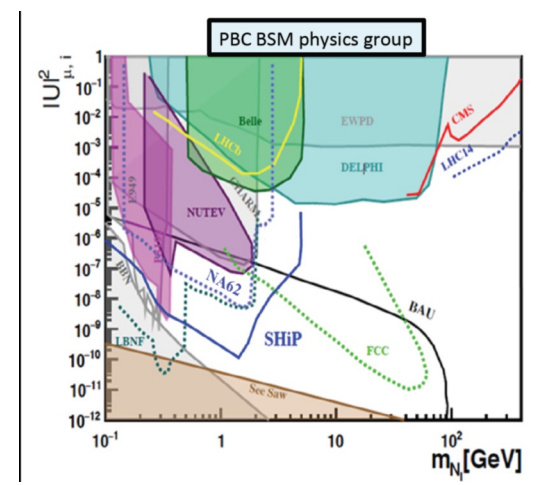

Figure 7. SHiP sensitivities to HNL (neutrino portal).

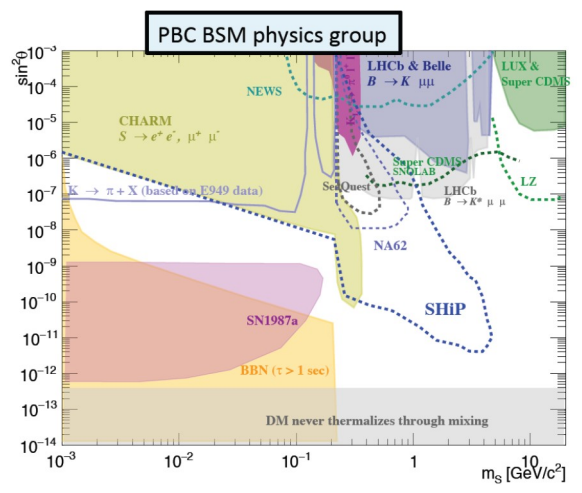

Figure 8. SHiP sensitivities to dark scalars (scalar portal).

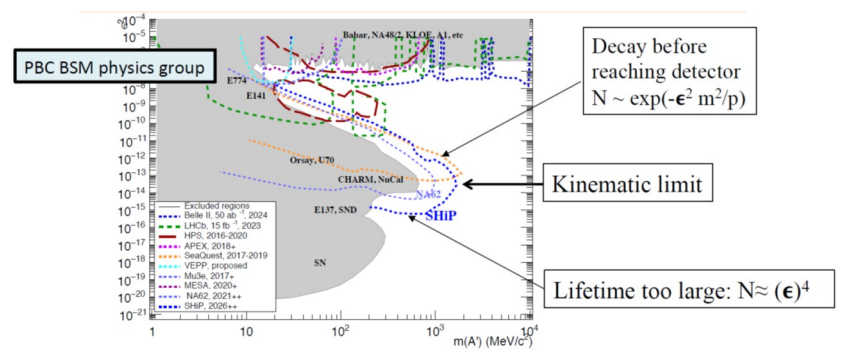

Figure 9. SHiP sensitivities to dark photons (vector portal).

eration of the SM as being the theory probably adequately describing physics up to the Planck scale. From experimental point of view, this can be interpreted as an argument in favor of NP (responsible, in particular, for neutrino oscillations and dark matter) manifested by relatively light, but very feebly interacting particles. The search of the latter should be done on intensity frontier rather than on energy frontier, and the proposed Search for Hidden Particles (SHiP) experiment at CERN's SPS, discussed in the present talk, is an example of this kind. 
Search for Hidden Particles (SHiP) experiment is complement to searches for New Physics at energy frontier at CERN. It is proposed to search for New Physics in the largely unexplored domain of new, very weakly interacting particles with masses $O(10) \mathrm{GeV} / \mathrm{c}^{2}$. It provides unique opportunities for tau-neutrino studies. Sensitivity improves previous experiments by factor $O\left(10^{4}\right)$ for Hidden Sector and by $O\left(10^{2}\right)$ for neutrino physics. The development of the project can be followed on [8].

\section{References}

[1] D. Buttazzo, G. Degrassi, P. P. Giardino, G. F. Giudice, F. Sala, A. Salvio and A. Strumia, JHEP 1312 (2013) 089, arXiv:1307.3536.

[2] C. D. Froggatt and H. B. Nielsen, Standard model criticality prediction: Top mass 173 +- 5-GeV and Higgs mass 135 +- 9-GeV, Phys. Lett. B 368 (1996) 96, hep-ph/9511371.

[3] T. Asaka, S. Blanchet and M. Shaposhnikov, The nuMSM, dark matter and neutrino masses, Phys. Lett. B 631 (2005) 151, hep-ph/0503065.

[4] S. Alekhin et al., A facility to Search for Hidden Particles at the CERN SPS: the SHiP physics case, Rept. Prog. Phys. 79 (2016) no.12, 124201, arXiv:1504.04855 [hep-ph].

[5] The SHiP Collaboration, A facility to Search for Hidden Particles (SHIP) at the CERN SPS, April 2015, arXiv:1504.04956.

[6] A. Akmete et al. [SHiP Collaboration], JINST 12 (2017) no.05, P05011 [arXiv:1703.03612 [physics.ins-det]].

[7] A.Golutvin, talk at the workshop ZPW2018 - Flavours: light, heavy and dark, University of Zurich, 15-17 January 2018; https://indico.cern.ch/event/667965/

[8] https://ship.web.cern.ch/ship/ 